\title{
A concise review of dynamical processes in polymorphic environments of a block copolymer: Rotational diffusion and photoisomerization
}

\author{
K S MALI and G B DUTT* \\ Radiation \& Photochemistry Division, Bhabha Atomic Research Centre, Trombay, Mumbai 400085 \\ e-mail: gbdutt@barc.gov.in
}

\begin{abstract}
This article describes our ongoing efforts to understand dynamical processes such as rotational diffusion and photoisomerization in polymorphic environments of a block copolymer. The objective is to explore how the typical properties of a block copolymer solution such as critical micelle temperature (CMT) and temperature-induced sol-gel transition influence the rotational diffusion of hydrophobic solute molecules. Rotational diffusion of solute molecules differs significantly below and above the CMT of a block copolymer solution, while there is no influence of sol-gel transition on solute rotation. This is rationalized on the basis of the site of solubilization of the solute molecules which is the palisade layer of the micelles in both phases and unaffected by gelation. A similar result has been obtained in case of photoisomerization studies carried out with a carbocyanine derivative in the sol and gel phases of the block copolymer. The isomerization studies have been extended to the reverse phases (sol and gel phases) of the block copolymer to explore the nature of the water present in the cores of the reverse micelles. Our results provide evidence for the existence of water droplets with properties resembling bulk water. In essence, we show that despite having vastly differing bulk properties, both the solution and gel phases (normal as well as reverse) offer identical microscopic environment.
\end{abstract}

Keywords. Block copolymer; critical micelle temperature; inverse melting transition; rotational diffusion; photoisomerization.

\section{Introduction}

The ability to display a rich structural polymorphism in addition to their widespread industrial applications has resulted in numerous investigations being carried out with water-soluble triblock copolymers in recent times. ${ }^{1-18}$ Almost all these investigations have dealt with the aggregation and phase behaviour of these systems. Typical triblock copolymers are composed of poly(ethylene oxide), (PEO) and poly (propylene oxide), (PPO) units and have the chemical composition $(\mathrm{PEO})_{x}-(\mathrm{PPO})_{y}-(\mathrm{PEO})_{x}$, where $x$ and $y$ represent the number of ethylene oxide and propylene oxide units, respectively. Unlike conventional surfactants, these triblock copolymers do not possess a polar head group and nonpolar tail, but attain amphiphilic character as a consequence of preferential solubility of one block over the other in a given solvent. For example, the PPO block is soluble in water below $288 \mathrm{~K}$, but turns hydrophobic at elevated temperatures because of its diminishing hydrogen-bonding interaction with water. In contrast, the PEO block is predominantly hydrophilic within

*For correspondence the temperature range $273-373 \mathrm{~K} .{ }^{6}$ However, it must be noted that solubility of the PEO block also decreases with temperature, but the effect is less pronounced compared to that of the PPO block. Due to the disparities in the relative solubilities of the two blocks, triblock copolymers form aggregates in water above a critical micelle temperature (CMT) and a critical micelle concentration (CMC) with a core consisting of predominantly PPO blocks and a corona made up of hydrated PEO blocks. The CMTs and CMCs of a number of aqueous triblock copolymers have been determined in a systematic manner by Alexandridis et $a l^{7}$ It is evident from their study that copolymers with larger number of PPO units form micelles at lower concentrations, and for a given copolymer concentration, they have lower CMTs. Other important micellar parameters such as aggregation number, hydrodynamic radius, and core radius are also available in the literature for the micelles formed with triblock copolymers in water. ${ }^{8,13}$ Since the solubility of PPO block decreases with temperature, depending on the concentration and temperature, these block copolymers exist as either unimers or micelles or even gels. It has been well established that at lower temperatures, 
the copolymers in water exist as Gaussian unimers, which upon increase in temperature aggregate to form micelles and a further raise in temperature results in gelation due to micelle-micelle entanglement. The temperature-induced sol-gel transition in these systems is known as inverse melting transition, ${ }^{3}$ and for gelation to occur the volume fraction of the micelles in solution should reach 0.52 , which implies that gelation is not possible in dilute block copolymer solutions. In these gels, spherical micelles are usually arranged in a cubic lattice. Details concerning the specific aspects of gelation for typical triblock copolymer systems have been discussed in literature. ${ }^{13}$

Apart from forming micelles, ternary systems consisting of copolymer-water-oil, depending on their composition, form many different phases. ${ }^{3,8,10,14}$ It has been established that besides the ternary composition, solvent polarity and block composition of the copolymer play a major role in the microstructure attained by these ternary systems. P123 is a typical triblock copolymer whose composition is $(\mathrm{PEO})_{20}-(\mathrm{PPO})_{70}-(\mathrm{PEO})_{20}$ and its phase behaviour has been investigated in detail by Holmqvist et $a l^{14}$ recently. Figure 1 gives the phase diagram of the ternary system P123-butyl acetate-water, which has been taken from ref. [14]. It is evident from the figure that in the ternary system of P123-butyl acetatewater, normal micellar $\left(L_{1}\right)$, normal micellar cubic $\left(I_{1}\right)$, reverse micellar $\left(L_{2}\right)$, and reverse micellar cubic $\left(I_{2}\right)$ phases are present, besides a variety of lyotropic

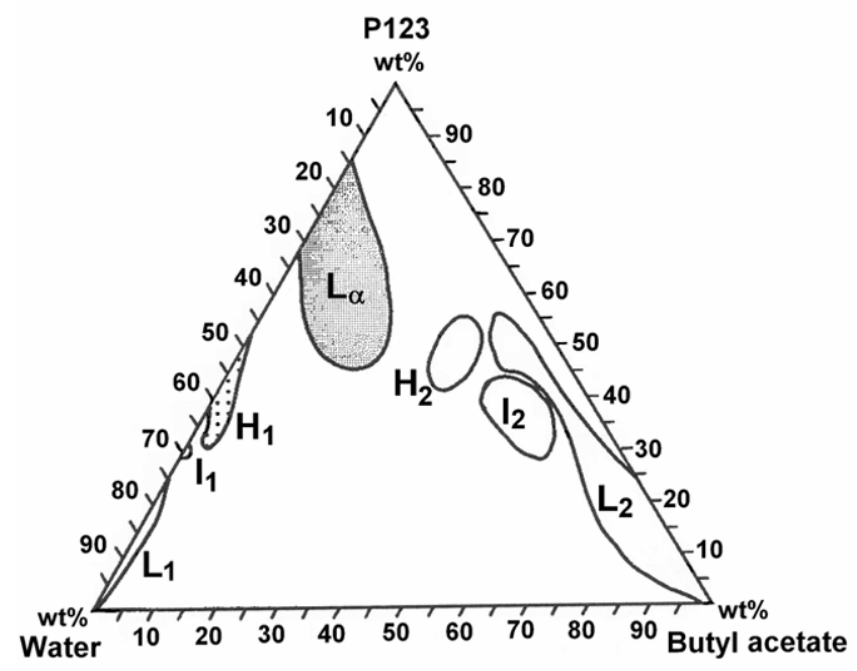

Figure 1. Phase diagram of P123-butyl acetate-water ternary system. Partially modified and reprinted with permission from ref. [14]. C) (1998) American Chemical Society. liquid crystalline phases. Although structural details such as aggregation number, hydrodynamic radius, core radius, and degree of hydration of the micelles present in three of the four phases are not available, the composition ranges of the three components for forming $L_{1}, I_{1}, L_{2}$ and $I_{2}$ phases have been established. In the $L_{1}$ phase, the concentration of P123 can go up to $25 \%$ and less than $5 \%$ of butyl acetate can be accommodated. In contrast, $I_{1}$ is formed around a narrow concentration region of $\mathrm{P} 123$, which is $30 \%$ and this phase can hold less than $2 \%$ of butyl acetate. In the $I_{1}$ phase, spherical micelles are arranged in a primitive cubic structure. The reverse micellar phase $L_{2}$ is formed with butyl acetate concentration as low as $40 \%$ and $\mathrm{P} 123$ up to $50 \%$, but can accommodate no more than $10 \%$ of water. In the concentration range of $30 \%-40 \% \mathrm{P} 123$ and $42 \%-58 \%$ butyl acetate, the reverse micellar cubic phase $I_{2}$ is formed, which can hold maximum water content of $10 \%-20 \%$. It has been established that in the $I_{2}$ region reverse micelles are arranged in a facecentred cubic structure. ${ }^{14}$

Even though considerable efforts have been directed towards elucidating the structures of these phases, attempts to understand dynamical processes such as rotational diffusion, ${ }^{19-24}$ solvation dynamics, ${ }^{25,26}$ and photoisomerization ${ }^{27-29}$ have only begun recently. These studies reveal information pertinent to the environment in the vicinity of the site of solubilization of the solute molecule and also enable us to comprehend these processes in confined systems. Understanding the microenvironment of these systems is imperative in view of their potential utility as microreactors for carrying out chemical reactions and as drug-delivery systems. To address these issues, we have initiated rotational diffusion ${ }^{20-22}$ and photoisomerization ${ }^{27-29}$ studies in polymorphic environments of a triblock copolymer, P123. Since these two dynamical processes are sensitive to the frictional coupling between the solute and the surrounding medium, the outcome of our work has enabled us to get a better appreciation of the microenvironment of these systems. The specific issues, which are discussed in this article, include how critical micelle temperature influences rotational diffusion of solute molecules and whether inverse melting transition or temperature-induced sol-gel transition has any effect on solute rotation. This article also discusses how the microviscosities of block copolymer micelles compare with that of nonionic micelles such as Triton X-100 (TX-100) and Brij35 , since the micelles formed with these two types 
of amphiphiles contain hydrated PEO chains as the corona or the palisade layer. Photoisomerization studies carried out in micellar $\left(L_{1}\right)$ and gel $\left(I_{1}\right)$ phases of P123 reveal if there are any differences in the isomerization rate constants in these two phases. Similar investigations undertaken in reverse micellar $\left(L_{2}\right)$ and reverse micellar gel phases $\left(I_{2}\right)$ shed light on the nature of the water present in these systems. We hope that the results presented in this article and the references given at the end will provide a glimpse of the recent work that is being carried out in block copolymer systems, especially from a photochemist's perspective. Finally, keeping in tune with the thematic nature of this special issue, the work described here has utilized time-resolved and steadystate fluorescence techniques to measure parameters such as fluorescence lifetimes, anisotropy decay constants and quantum yields.

\section{Rotational diffusion studies}

Rotational diffusion studies involving medium-sized solute molecules in homogeneous media have often been employed to explore solute-solvents interactions. ${ }^{30,31}$ In contrast, such investigations in microheterogeneous systems have enabled us to understand the dynamics in confined systems and also provided valuable information pertinent to their microenvironments. In recent times, numerous studies have been undertaken in micelles ${ }^{32-40}$ and reverse micelles ${ }^{41-43}$ to achieve this objective. In the present work, our aim is to extend these studies to aggregates formed with block copolymers. For this purpose, two structurally similar hydrophobic probes, 2,5dimethyl-1,4-dioxo-3,6-diphenylpyrrolo[3,4-c]pyrrole (DMDPP) and 1,4-dioxo-3,6-diphenylpyrrolo[3,4c]pyrrole (DPP) have been employed. The molecular structures of DMDPP and DPP are given in figure 2 and these probes have been chosen because of the following reasons. The rotational diffusions of DMDPP and DPP have been extensively investigated in homogeneous solutions, ${ }^{30}$ nonionic micelles, ${ }^{35-37}$ nonionic reverse micelles, ${ }^{42,43}$ and also in ionic liquids. ${ }^{44}$ Even though both DMDPP and DPP are structurally similar, their chemical nature is somewhat different. DPP because of the presence of two secondary amino groups forms strong hydrogen bonds with the hydroxyl, carbonyl, sulphoxide and alkoxy groups of the solvents and as a consequence its rotational diffusion is much slower compared to DMDPP. These probes because of their hydrophobic nature are located in the micellar phase rather than the aqueous phase. The relatively long fluorescence lifetimes (6-8 ns) of both DMDPP and DPP enable us to recover long-time constants associated with the anisotropy decays in block copolymer micelles.

\subsection{Influence of critical micelle temperature on solute rotation}

Rotational diffusions of DMDPP and DPP (the concentrations of the probes used are in the region of $10^{-5}-10^{-6} \mathrm{M}$ ) has been examined in $1 \% \mathrm{w} / \mathrm{v}$ aqueous solution of P123 over the temperature range 283$318 \mathrm{~K}$ in steps of $5 \mathrm{~K}$. The objective is to measure anisotropy decays of the probes below and above the CMT. It must be noted that the CMT of a block copolymer solution decreases with an increase in the concentration of the copolymer. In fact, data available for a number of triblock copolymers in literature $^{7}$ indicate that there is a linear relationship between CMT and $\log$ [copolymer], and figure 3 displays such a plot for P123. It is evident from the figure that the CMT of $1 \% \mathrm{w} / \mathrm{v}$ aqueous $\mathrm{P} 123$ is $289 \mathrm{~K}$. In other words, $1 \%$ solution of P123 in water forms micelles above $289 \mathrm{~K}$ and below this temperature only unimers of P123 are present. Typical anisotropy decays of DMDPP and DPP measured in 1\% $\mathrm{P} 123$ at $283 \mathrm{~K}$ and $318 \mathrm{~K}$ are given in figure 4 . The interesting aspect to be noted from the figure is that anisotropy decays at $318 \mathrm{~K}$ are significantly slower compared to the ones measured at $283 \mathrm{~K}$ despite an increase in temperature of the solution by $25 \mathrm{~K}$. Moreover, anisotropy decays of DMDPP and DPP can be fit with single exponential functions below CMT. In contrast, above CMT, biexponential functions with two time constants are needed to fit the anisotropy decays and the functional form is given by

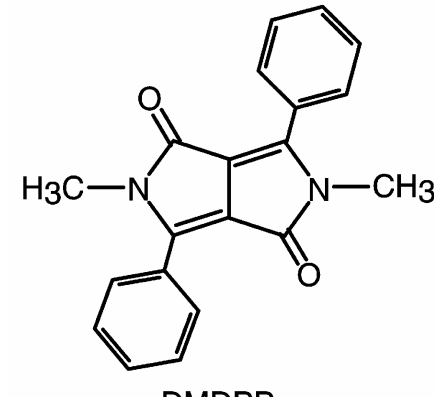

DMDPP

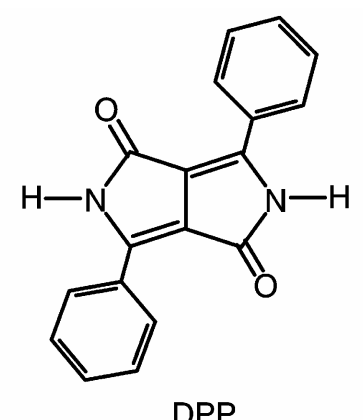

DPP
Figure 2. Molecular structures of DMDPP and DPP. 


$$
r(t)=r_{0}\left[\beta \exp \left(-t / \tau_{1}\right)+(1-\beta) \exp \left(-t / \tau_{2}\right)\right] .
$$

In the above equation, $r_{0}$ is the limiting anisotropy of the solute, whose magnitude is dictated by the angle between the absorption and emission transition dipoles, $\tau_{1}$ and $\tau_{2}$ are the long and short components associated with the decay of anisotropy of the solute, respectively and $\beta$ is the percentage contribution of $\tau_{1}$. To compare the rotational diffusion of the probes below and above CMT, we have calculated the average reorientation time $\left\langle\tau_{r}\right\rangle$ from $\tau_{1}$ and $\tau_{2}$ using

$$
\left\langle\tau_{r}\right\rangle=\beta \tau_{1}+(1-\beta) \tau_{2}
$$

The $\left\langle\tau_{r}\right\rangle$ values of DMDPP and DPP are plotted as a function of temperature in figure 5. It is evident from the figure that variation of $\left\langle\tau_{r}\right\rangle$ with temperature is not uniform and there is a discontinuity in the plots at $289 \mathrm{~K}$. This pattern can be comprehended by considering the rotational diffusion of the solute below and above the CMT of the copolymer solution.

2.1a Rotational diffusion below CMT: Since P123 exists as individual unimers in water below CMT, the rotational diffusion of the probes under these conditions should in principle be similar to that observed in a number of homogeneous solvents. ${ }^{30}$ The reorientation times of DMDPP are 0.32 and $0.29 \mathrm{~ns}$

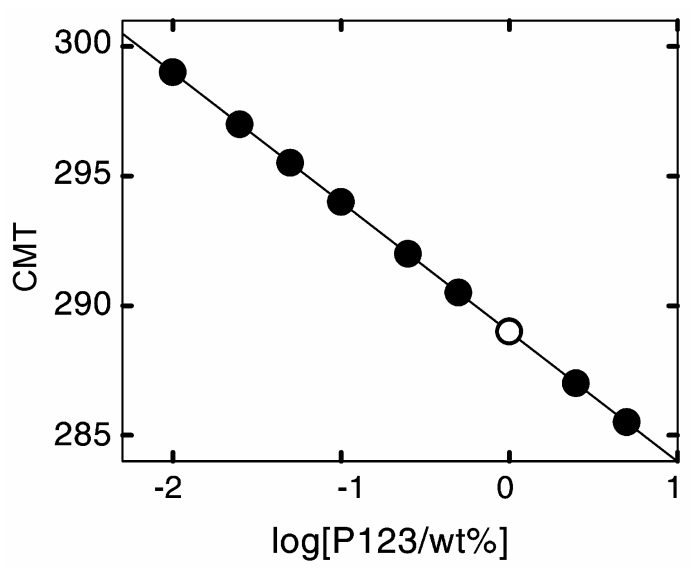

Figure 3. Variation of CMT as a function of $\log [\mathrm{P} 123]$ and smooth line passing through the data points is obtained by linear least-squares fit. The abscissa of the open circle shown in the figure represents the concentration of the copolymer at which rotational diffusion measurements have been performed and the ordinate gives the corresponding CMT. The data presented in this figure are reproduced with permission from ref. [7]. (C) (1994) American Chemical Society. at 283 and $288 \mathrm{~K}$, respectively. It has also been noticed that the rotation of DMDPP is faster than DPP by over a factor of four at 283 as well as $288 \mathrm{~K}$. One of the reasons for such behaviour is the presence of strong hydrogen-bonding interactions of DPP through its secondary amino groups with the oxygen atoms of the PEO units. Now, we are interested in comparing the reorientation times of DMDPP with the ones calculated using the Stokes-Einstein-Debye (SED) hydrodynamic theory. ${ }^{45}$ Since DMDPP is a medium-sized solute molecule that does not strongly interact with most of the solvents, ${ }^{30}$ its rotational
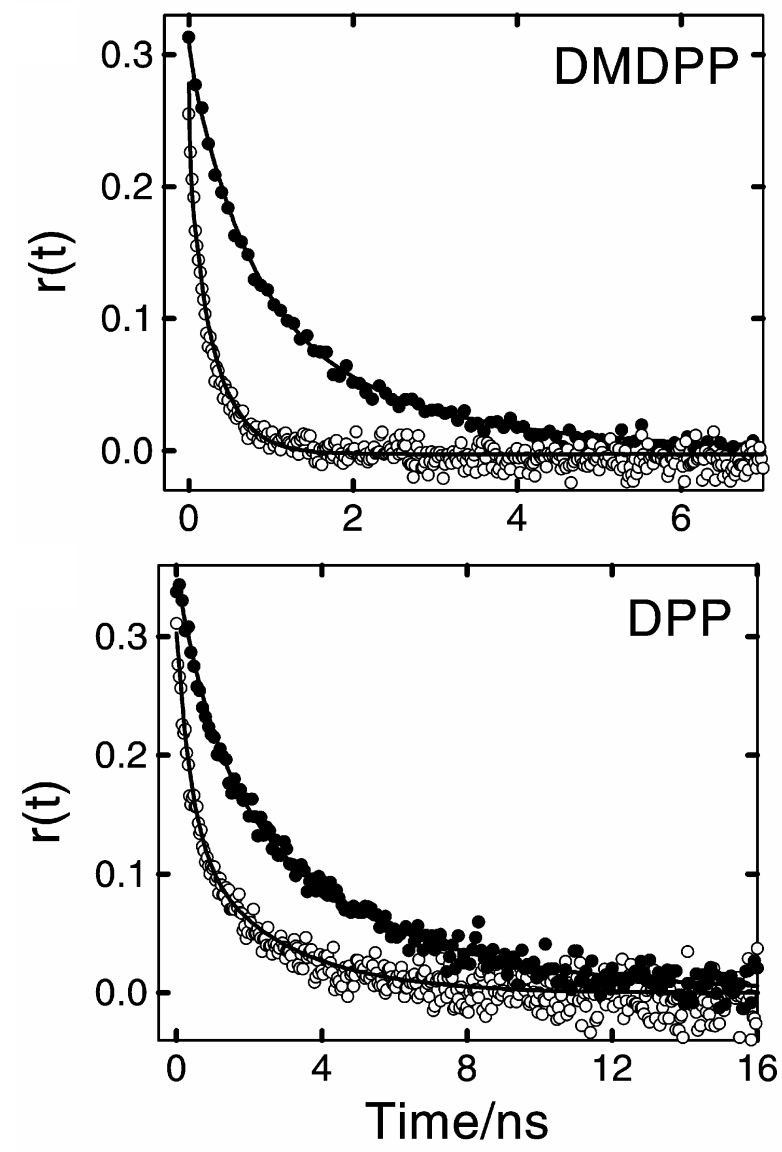

Figure 4. Anisotropy decays of DMDPP and DPP in aqueous solutions of P123 at $283 \mathrm{~K}(\mathrm{O})$ and $318 \mathrm{~K}\left(\mathrm{O}_{\text {). }}\right.$ Notice that faster anisotropy decays have been observed for DMDPP as well as DPP at lower temperature. These decays have been measured below and above the critical micellar temperatures. The smooth lines passing through them are the fitted ones. Below CMT, the reorientation times of DMDPP and DPP are $0.32 \pm 0.03$ and $1.4 \pm$ $0.1 \mathrm{~ns}$, respectively, at $283 \mathrm{~K}$. Above CMT, the average reorientation times of DMDPP and DPP are $1.3 \pm 0.2 \mathrm{~ns}$ and $2.9 \pm 0.2 \mathrm{~ns}$, respectively, at $318 \mathrm{~K}$. Reprinted with permission from ref. [20]. C (2005) American Chemical Society. 
diffusion has adequately been described by the SED theory with slip-boundary condition. ${ }^{46}$ The calculated reorientation times with slip- and stick-boundary conditions at unit viscosity $\eta$ and temperature $T$ that have been taken from our earlier work ${ }^{47}$ are $13.18 \times \eta / T$ and $41.41 \times \eta / T \mathrm{~ns} \mathrm{~K}\left(\mathrm{mPa} \mathrm{s}^{-1}\right.$, respectively. A comparison between the experimentally measured reorientation times and the theoretically calculated numbers with stick-boundary condition indicates that the rotation of DMDPP is slower by a factor of $1.7-1 \cdot 8$, which is very slow compared to its rotation in a number of solvents. ${ }^{30}$ What is the reason for such a slow rotation of DMDPP in aqueous solutions of P123? The rationale being the hydrophobic nature of the probe, which prevents it from experiencing the bulk viscosity of the medium, and as a consequence, the probe molecule is engulfed by the copolymer. In other words, the microenvironment offered by the copolymer surroundings is much less fluid than the macroscopic viscosity of water even in the absence of specific interactions. If DMDPP were to experience aqueous surroundings its reorientation times would have been 142 and 123 ps at 283 and $288 \mathrm{~K}$, respectively, based on its measured value in water at $298 \mathrm{~K}$. In case of DPP, however, the presence of specific interactions between the probe and the surroundings impede its rotation significantly compared to DMDPP. It has been demonstrated in our earlier work ${ }^{30}$ that the degree of slowness of the rotation of DPP compared to DMDPP, which is in the range of $1.5-3.0$ in numerous solvents, depends

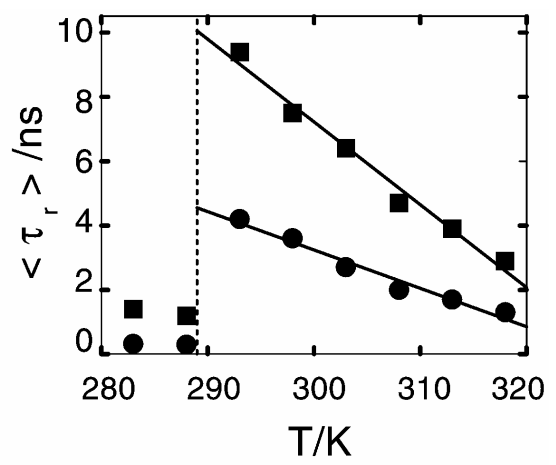

Figure 5. Plots of average reorientation times of DMDPP (O) and DPP ( $\mathbf{\square}$ ) in 1\% w/v aqueous P123 as a function of temperature. The vertical line (dotted line) in the figure represents the critical micelle temperature for this system. The lines passing through the data points have been obtained by linear least-squares fits. The two points below the CMT are not considered for the fit. The data presented in this figure are reproduced with permission from ref. [20]. C) (2005) American Chemical Society. on the strength of the interaction between the solute and the solvent. Nonetheless, in the present system this factor exceeds four, which is an indication that DPP not only experiences specific interactions but also resides in a different location of the copolymer compared to DMDPP; a result similar to that observed in reverse micelles of TX-100. ${ }^{42,43}$

2.1b Rotational diffusion above CMT: The copolymer, P123, associates and forms spherical micelles above CMT, and hence, the rotational diffusion of the probes is quite different compared to that observed below CMT. The aggregation number $N_{\text {agg, }}$, hydrodynamic radius $r_{h}$ and core radius $r_{c}$ of these micelles as a function of temperature, which have been obtained from the literature, ${ }^{8,13}$ are given in table 1. It is evident from the table that with increase in temperature, the aggregation number of these micelles goes up. However, the hydrodynamic radius remains more or less the same especially from $303-318 \mathrm{~K}$. This observation has been rationalized on the basis of dehydration of PEO blocks at higher temperatures resulting in almost identical coronal volume. ${ }^{13}$

The biexponential behaviour observed above CMT can arise due to either the partitioning of the probe molecule between two different environments in micellar medium or the different kinds of motion experienced by it. To ascertain the proper reason for the observed behaviour, the location of the probe molecule must be established. Since the probes used in the present study DMDPP and DPP are hydrophobic they are not expected to be located in water. However, DMDPP is sparingly soluble in water and has a lifetime of $7.8 \mathrm{~ns}$ and reorientation time of 92 ps at $298 \mathrm{~K} .{ }^{20}$ On the contrary, in P123 micelles, the measured lifetime and the short component of the anisotropy decay $\left(\tau_{2}\right)$ are $8.6 \mathrm{~ns}$ and $1.0 \mathrm{~ns}$, respectively, at this temperature. In case of DPP, no fluorescence signal could be observed in water. The

Table 1. Properties of P123 micelles as a function of temperature. ${ }^{\text {a }}$

\begin{tabular}{lrcc}
\hline Temperature $(\mathrm{K})$ & $N_{\mathrm{agg}}$ & $r_{C}(\mathrm{~nm})$ & $r_{h}(\mathrm{~nm})$ \\
\hline 298 & 86 & $5 \cdot 2$ & $5 \cdot 77$ \\
308 & 244 & $7 \cdot 3$ & $8 \cdot 18$ \\
313 & 287 & $7 \cdot 7$ & $8 \cdot 63$ \\
318 & 297 & $7 \cdot 8$ & $7 \cdot 73$ \\
\hline
\end{tabular}

${ }^{a}$ Data presented in this table have been taken from refs [8] and [13] 
arguments presented here rule out the possibility that the two anisotropy decay components arise due to fraction of the solutes being solubilized in the aqueous phase surrounding P123 micelles. Having established that both DMDPP and DPP reside in the micellar phase, it has how to be ascertained whether they are solubilized in the palisade layer or in the core of these micelles. From our earlier work ${ }^{35-37}$ involving the rotational diffusion of these probes in nonionic micelles such as TX-100 and Brij-35, it has been established that they are located in the palisade layer of the micelles, which is essentially made up of PEO units. We arrived at this conclusion based on the fluorescence lifetime data for DMDPP and solubility criteria for DPP. The fluorescence lifetimes of DMDPP in nonpolar solvents such as $n$-hexane, cyclohexane, hexadecane, benzene and squalane are $5.58,6 \cdot 04,6.59,6.12$ and $6.71 \mathrm{~ns}$, respectively, at $298 \mathrm{~K}^{42,43,48}$ whereas the $\tau_{f}$ values of DMDPP in TX-100 and Brij-35 micelles are in the range of 8.3$8.70 \mathrm{~ns}$. These data clearly indicate that even though DMDPP is soluble in alkane-like solvents, it is not solubilized in the cores of TX-100 and Brij-35 micelles, which consist of nonpolar alkyl chains. In case of DPP, the evidence is much simpler to deduce, as it is not soluble in alkane-like environments. Moreover, extensive investigations carried out in the literature ${ }^{49-52}$ pertinent to the sites of solubilization of organic molecules in micelles indicate that they are indeed solubilized at or near the micelle-water interface. Based on the conclusions available in the literature and also from our earlier experience ${ }^{35-37}$ with the probes DMDPP and DPP in TX-100 and Brij-35 micelles, it is only reasonable to conclude that both the probes are solubilized in the palisade layer of the P123 micelles.

By now it has been well established that aromatic probes, which reside at or near the interface, undergo a slow lateral diffusion on or inside the curved surface of the micelle and also a fast wobbling motion in an imaginary cone. These two motions are coupled to the overall rotation of the micelle, which is known as the two-step model ${ }^{53-56}$ This model has been applied successfully to explain the rotational diffusion of organic solutes in confined systems such as micelles, ${ }^{32-40}$ reverse micelles, ${ }^{41-43}$ thermosensitive core-shell latex particles ${ }^{57}$ and polymersurfactant aggregates. ${ }^{58}$ The two-step model has been used to obtain lateral, wobbling diffusion coefficients, order parameters and cone angles in the present case as well. ${ }^{20}$ Since our main focus here is to compare the rotational diffusion of DMDPP and
DPP below and above the CMT of $1 \%$ aqueous $\mathrm{P} 123$, we refrain from further discussion on the twostep model.

\subsection{Effect of inverse melting transition on solute rotation}

As mentioned earlier, for gelation to occur the micellar volume fraction in solution should reach a value of 0.52 and, for this to happen, the copolymer concentration should be reasonably high. In the case of $\mathrm{P} 123,30 \% \mathrm{w} / \mathrm{v}$ aqueous solution of the copolymer turns into gel at $289 \mathrm{~K}^{18}$ When this type of sol-gel transition takes place, the viscosity of the solution increases significantly and it ceases to flow. Our interest is to determine how such inverse melting transition influences solute rotation and to this effect, we have examined the rotational diffusion of DMDPP and DPP in $30 \% \mathrm{P} 123$ over the temperature range $285-313 \mathrm{~K}$. The anisotropy decays of both the probes exhibit bi-exponential behaviour and the average reorientation times are plotted as a function of temperature in figure 6 . It can be noticed from the figure that there is no discontinuity in the plot near the sol-gel transition temperature for both the solutes. Since the variation in the measured parameters

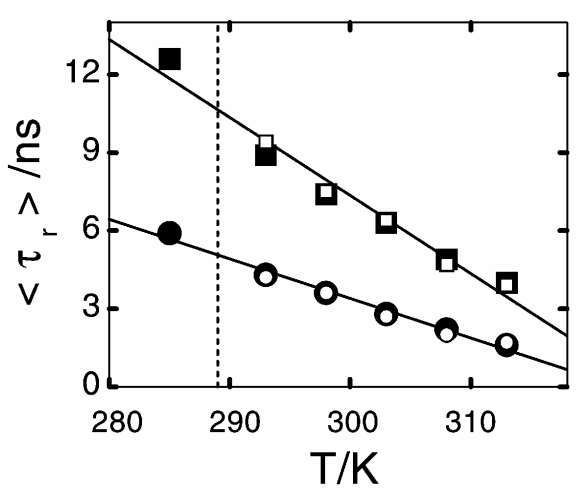

Figure 6. Plots of average reorientation times of DMDPP (O) and DPP (ם) in $30 \% \mathrm{w} / \mathrm{v}$ P123 as a function of $T$. The lines passing through the data points have been obtained by linear least squares fit. Notice that there is no abrupt change in $\left\langle\tau_{r}\right\rangle$ for both the solutes as the system undergoes sol-gel transition. The vertical line (dotted line) in the figure represents sol-gel transition temperature for $30 \% \mathrm{w} / \mathrm{v} \mathrm{P} 123$. It must be noted that the CMT for $30 \% \mathrm{w} / \mathrm{v} \mathrm{P} 123$ is about $281.6 \mathrm{~K}$. The open symbols are the corresponding average reorientation times of the solutes in $1 \% \mathrm{w} / \mathrm{v}$ P123. Incidentally, the dotted line also represents the CMT for $1 \%$ w/v P123. Partially modified and reprinted with permission from ref. [21]. (C) (2005) American Institute of Physics. 
is uniform with temperature, the data presented here do not reflect the phase transition experienced by the system. What is the reason for the observed behaviour? Even though there is a significant enhancement in the macroscopic viscosity of the system upon gelation, at the microscopic level, the gel is essentially made up of a high concentration of micelles in which the probe molecules are located. Since gelation results as a consequence of micellemicelle entanglement, the microenvironment of the interfacial region is affected, whereas that of the palisade layer remains the same. It may be recalled that DMDPP and DPP are solubilized in the palisade layer of the micelles in the sol as well as gel phases, thus the rotational diffusion of the probes is not influenced by the phase transition undergone by the copolymer solution. In other words, there is no change in the microviscosity of the palisade layer region of the micelles in both the phases. Thus, it would be interesting to compare the average reorientation times of the solutes with those measured in $1 \% \mathrm{w} / \mathrm{v}$ aqueous solution of P123. For this purpose, average reorientation times of DMDPP and DPP in $1 \%$ copolymer solution over the temperature range $293-313 \mathrm{~K}$ are also plotted in figure 6 . There is almost an identical match between the $\left\langle\tau_{r}\right\rangle$ values measured in $1 \%$ as well as $30 \% \mathrm{w} / \mathrm{v}$ P123 for both the probes. This result conclusively demonstrates that when a micellar solution transforms into gel, its macroscopic properties are altered significantly, whereas its microenvironment remains the same.

\subsection{Comparison of the microviscosities of P123 with other nonionic micelles}

At this juncture, it would be interesting to compare the microviscosities of P123 micelles with other nonionic micelles formed with TX-100 and Brij-35 surfactants, since the outer shell of all the three micelles comprises PEO units, the differences being the number of PEO units and the degree of hydration. The amphiphiles P123, TX-100, and Brij-35 have 20, 9-10, and 23 PEO units, respectively. Moreover, the probes are solubilized in the palisade layer region containing PEO units in all the three micellar systems. For comparison of microenvironments, average reorientation times of DMDPP in P $123,{ }^{20} \mathrm{TX}-100^{35}$ and Brij- $35^{37}$ have been employed. This is due the fact that DMDPP does not strongly interact with the surroundings and as a consequence its hydrodynamic volume remains the same from system to system; this aspect has been thoroughly discussed in our earlier papers. ${ }^{37-39}$ It has been found that a hydrodynamic volume $V_{h}$ of $183 \AA^{3}$ for DMDPP ${ }^{47}$ which is calculated with slip-boundary condition by treating the solute as an asymmetric ellipsoid, adequately accounts for its rotation in a number of solvent systems. ${ }^{30}$ The same value has been used to calculate the microviscosities $\left(\eta_{m}\right)$ of micelles ${ }^{37-39}$ with the aid of the SED equation, ${ }^{45}$

$$
\eta_{m}=\left\langle\tau_{r}\right\rangle_{P} k T / V_{h}
$$

In the above equation, $\left\langle\tau_{r}\right\rangle_{p}$ is the average reorientation time of the probe without the contribution from the overall rotation of the micelle. However, in case of large nonionic micelles such as P123, TX-100 and Brij-35, $\left\langle\tau_{r}\right\rangle_{p}$ and $\left\langle\tau_{r}\right\rangle$ are more or less the same, since the contribution from the overall rotation of the micelle to fluorescence depolarization of the probe is negligible. The microviscosities obtained in this manner for the three micelles as a function of temperature are presented in figure 7 . It is evident from the figure that $\eta_{m}$ values of P123 micelles are higher by factors of $1.2-1.3$ and 3.3-4.5 compared to TX-100 and Brij-35 micelles, respectively. This result clearly indicates that the magnitude of $\eta_{m}$ not only depends on the number of PEO units but also on the hydration levels. From the microviscosity data, we can conclude that the hydration levels of TX-100 and P123 micelles are somewhat similar but are significantly lower compared Brij-35 micelles.

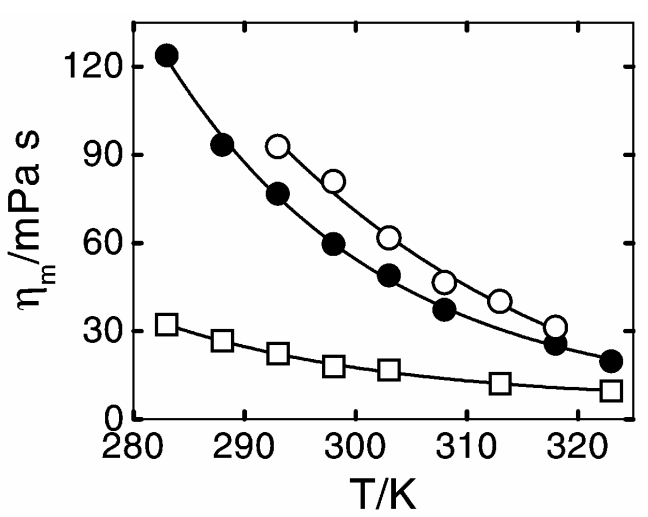

Figure 7. Plots of microviscosities of the micelles P123 (O), TX-100 (०), and Brij-35 ( $\square$ ) obtained from the average reorientation times of the probe DMDPP. The smooth lines through the data points have been obtained by fitting them to exponential functions. Reprinted with permission from ref. [20]. C (2005) American Chemical Society. 


\section{Photoisomerization studies}

The photoisomerization of olefins and polyenes has been extensively investigated in the recent past as prototypical examples of unimolecular reactions in condensed phase. ${ }^{31,59,60}$ The excited state isomerization of these systems involves an activated twist motion about the double bond/bonds to an intermediate geometry. In the twisted geometry, the excited state becomes close in energy to the ground state, thus it rapidly decays to the twisted ground electronic state by internal conversion. Once in the ground state surface, branching between the isomer and the normal form occurs. The nonradiative process is dominated by the barrier crossing or the twisting motion because the internal conversion from the twisted excited state is very rapid. Since a large amplitude motion is involved in the barrier crossing, the nonradiative rate depends on solute-solvent frictional coupling. Moreover, because of the polar nature of the transition state solute-solvent dielectric interactions also govern the isomerization rate. Kramer's theory ${ }^{61}$ has been widely applied to understand the isomerization rates in terms of barrier heights. ${ }^{31,59,60}$ Although Kramer's theory has been quite successful, systematic deviations from Kramer's equation have been observed, which were rationalized on the basis of microscopic friction being different from the bulk viscosity of the solvent, frequency dependent frictional effects and inadequacy of the one-dimensional model. Among the systems investigated include stilbene and its analogues, ${ }^{60}$ diphenylbutadienes ${ }^{62-67}$ and carbocyanines. ${ }^{68-73}$

Even though extensive studies have been carried out to understand the photoisomerization in liquids, only a few reports are available in literature that deal with the isomerization at surfaces and interfaces. ${ }^{74-77}$ Sitzmann and Eisenthal ${ }^{74}$ have investigated the photoisomerization of 3,3'-diethyloxadicarbocyanine iodide (DODCI) at the air-water interface using surface second harmonic generation techniques and found that the isomerization rate is significantly faster compared to bulk water due to the reduced friction at the interface. On the other hand, Bhattacharyya and co-workers ${ }^{76,77}$ have studied the photoisomerization of DODCI in micelles and reverse micelles and observed an opposite trend as a consequence of the probe experiencing increased friction at the micelle-water interface compared to bulk water. In the present work, photoisomerization studies have been carried out in normal and reverse phases of P123 as a function of temperature. For this purpose we have used the cationic probe DODCI (see figure 8 for the molecular structure) since it is expected to be located at the interface in case of normal phases and in the core region in the reverse phases.

\subsection{Photoisomerization of DODCI in $L_{1}$ and $I_{1}$ phases}

The aim of this work is to find out whether the isomerization rate constants of DODCI are identical or not in aqueous block copolymer micellar solutions and gels and also how they compare to that in aqueous medium. For this purpose, we have measured the fluorescence quantum yields $\left(\phi_{f}\right)$ and lifetimes of DODCI in water, $5 \%\left(L_{1}\right)$ and $30 \%\left(I_{1}\right)$ aqueous P123 solutions over the temperature range 293$318 \mathrm{~K}$. The concentration of DODCI used in these experiments is about $10 \mu \mathrm{M}$. Figure 9 gives plots of $\phi_{f}$ vs $T$ for DODCI in water, $L_{1}$ and $I_{1}$ phases of $\mathrm{P} 123$. It is evident from the figure that there are no significant differences in the measured quantum yields of DODCI in $L_{1}$ and $I_{1}$ phases. In contrast, the corresponding numbers in water are considerably lower. This result is not surprising considering the

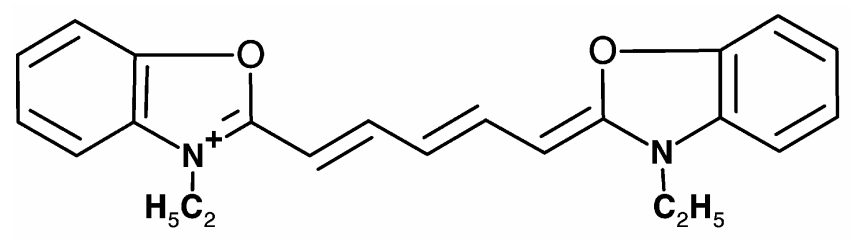

Figure 8. Molecular structure of DODCI.

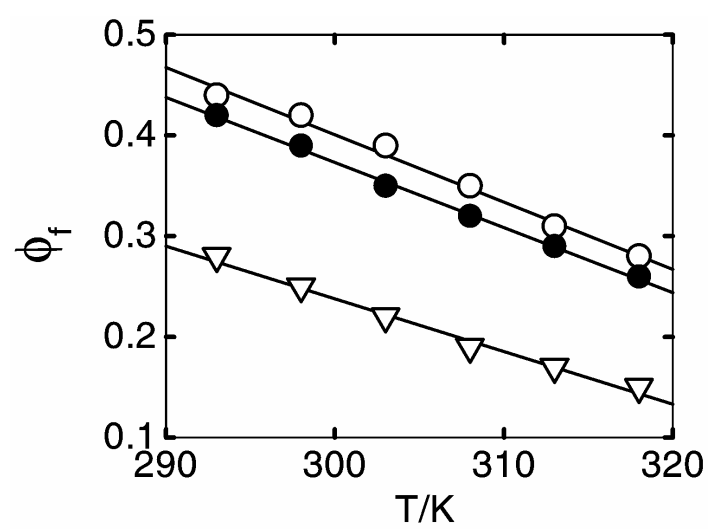

Figure 9. Plots of quantum yields vs $T$ for DODCI in water $(\nabla), 5 \% \mathrm{P} 123(\bigcirc)$, and $30 \% \mathrm{P} 123(\mathrm{O})$. The lines passing through the data points have been obtained by linear least-squares fit. Reprinted with permission from ref. [27]. C (2006) American Institute of Physics. 
fact that isomerization rates in water are expected to be appreciably faster than in the micellar media. Fluorescence decays $(f(t))$ of DODCI in water could be fit with single exponential function over the entire temperature range studied. On the contrary, these decays in 5\% and 30\% P123 follow a bi-exponential function described by the following equation.

$$
f(t)=a \exp \left(-t / \tau_{f 1}\right)+(1-a) \exp \left(-t / \tau_{f 2}\right) .
$$

In the above equation, $\tau_{f 1}$ and $\tau_{f 2}$ are the two lifetimes associated with the decay of fluorescence and $a$ is the percentage contribution of $\tau_{f 1}$ to the decay. It has been noticed that the recovered values of $\tau_{f 2}$ in both the phases are identical to the ones measured in water and the contribution of this component is only $20-30 \%$. This result indicates that a small fraction of DODCI is located in aqueous phase surrounding the micelles in $L_{1}$ as well as $I_{1}$ phases of P123. However, the surprising observation from these measurements is that the magnitudes of the major component are also identical in both the phases and it has been noticed that the values of $\tau_{f 1}$ are longer than $\tau_{f 2}$ by a factor of $2 \cdot 5$.

From the rotational diffusion results presented earlier, it is evident that the microenvironment of the palisade layer is the same in $L_{1}$ and $I_{1}$ phases. Since DODCI is an isomerization probe, its lifetimes are sensitive to the local environment. However, experimental data indicates that the longer component $\left(\tau_{f 1}\right)$ of DODCI at a given temperature in 5\% and 30\% aqueous P123 is identical, which is possible only if the probe is solubilized in palisade layer. On the other hand, if the probe is located at the interface, its lifetimes in both the phases would have been different. This is due to the fact that the friction experienced by the solute molecule in the interfacial region increases with micellar concentration as a consequence of interpenetrating PEO chains. ${ }^{22}$ This result is somewhat unexpected considering the fact that DODCI is a charged solute and is more likely to be solubilized at the interface where the concentration of the water is high. However, the PEO chains that constitute the palisade layers of nonionic micelles and micelles formed with block copolymers are hydrated and contain significant amounts of water. The water present in the palisade layer is either thermodynamically bound to the PEO chains or mechanically trapped inside the meandering PEO units. ${ }^{78,79}$

The nonradiative rate of DODCI has been identified as the rate of isomerization about the double bond. ${ }^{70}$ Hence, to comprehend the isomerization process in these systems, nonradiative rates have been obtained using procedure described below. Fluorescence quantum yield and lifetime are related to radiative $\left(k_{r}\right)$ and nonradiative $\left(k_{n r}\right)$ rate constants by the following relations, ${ }^{80}$

$$
\begin{gathered}
\phi_{f}=k_{r} /\left(k_{r}+k_{n r}\right), \\
\tau_{f}=1 /\left(k_{r}+k_{n r}\right) .
\end{gathered}
$$

Due to the partitioning of the probe between micellar and aqueous phases, the quantum yield of the probe partitioned in the micellar phase $\left(\phi_{\text {micelle }}\right)$ has been obtained from the measured quantum yield in aqueous P123 $\left(\phi_{P 123}\right)$ and water $\left(\phi_{\text {water }}\right)$ using the following relation,

$$
\phi_{P 123}=\left[\frac{a \tau_{f 1} \phi_{\text {micelle }}+(1-a) \tau_{f 2} \phi_{\text {water }}}{a \tau_{f 1}+(1-a) \tau_{f 2}}\right] .
$$

The basis for employing (7) to obtain quantum yields of the probe located in different regions of the microheterogeneous system is as follows. Since quantum yield is independent of emission wavelength, the observed fluorescence decay has been integrated over the entire emission spectrum to obtain appropriate weights for $\phi_{\text {micelle }}$ and $\phi_{\text {water }}$. From the parameters $\phi_{\text {micelle }}$ and $\tau_{f 1}$, the radiative and nonradiative rate constants of the fraction of DODCI located in the palisade layer of the micelles for 5\%

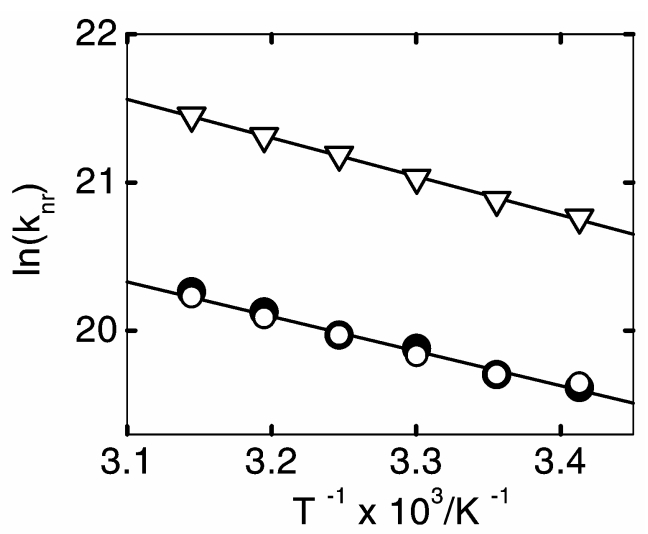

Figure 10. Plots of $\ln \left(k_{n r}\right)$ vs $1 / T$ for DODCI in water $(\nabla), 5 \%(\bigcirc)$ and $30 \%$ aqueous P123 (O). The lines passing through the data points have been obtained by linear least-squares fit. Reprinted with permission from ref. [28]. C) (2006) American Institute of Physics. 
and $30 \%$ aqueous P123 have been obtained. Since the variation of photoisomerization rate constant with temperature follows Arrhenius relation, the parameters $\ln \left(k_{n r}\right)$ and $1 / T$ have been plotted as ordinate and abscissa, respectively, in figure 10 for DODCI in water, $5 \%$ and $30 \%$ aqueous P123. It is evident from the figure that the isomerization rate constants in $L_{1}$ and $I_{1}$ phases of P123 are identical but are significantly lower compared to that in water. The result obtained here is explicable considering the fact that organized media offer more friction for processes such as photoisomerization compared to homogeneous systems. Moreover, the identical isomerization rate constants obtained in micellar solution and gel phases, complement our rotational diffusion results.

\subsection{Photoisomerization of DODCI in $\mathrm{L}_{2}$ and $\mathrm{I}_{2}$ phases}

From the photoisomerization studies carried out in $L_{1}$ and $I_{1}$ phases of P123 it has become evident that DODCI is distributed between aqueous and micellar phases. This evidence has provided impetus to use DODCI as a probe to examine nature of water present in the reverse phases of P123. Unlike the reverse micelles formed with ionic surfactants, where well-defined and well-characterized water droplets exist over a wide composition range, the characteristics and constitution of water present in nonionic reverse micelles are not thoroughly understood. This is due to the fact that hydration of the polyethylene oxide head groups and the subsequent formation of the water droplet depend on the polarity of the oil and also its ability to penetrate inside the polar cores. The nonionic surfactant, TX-100 is one such example and it forms reverse micelles in cyclohexane ${ }^{81,82}$ mixed solvents of benzene- $n$-hexane ${ }^{83,84}$ and in toluene ${ }^{85,86}$ under certain conditions. It has been entrenched that some of these systems form water droplets above a certain value of the mole ratio of water to surfactant $(W)$, while in other systems the added water merely hydrates the polyethylene oxide head groups. Even though it has been established that the copolymer P123 forms reverse phases $\left(L_{2}\right.$ and $\left.I_{2}\right)$, it is not known whether the water present in these systems is in the form of droplets and these droplets have the properties of bulk water. The present study has been undertaken to address this issue and also investigate the photoisomerization process in the reverse phases.
In the preparation of these reverse phases, $n$-butyl acetate was used as the nonpolar component or the oil. The chosen weight ratios of P123-butyl acetatewater are $14: 80: 6,36: 58: 6$, and $36: 52: 12$. The first two are the reverse micellar phases and can designated as $L_{2}$ and ${ }^{1} L_{2}$, respectively, whereas the third one is a reverse micellar cubic phase, $I_{2}$ and the $W$ values for the three systems are 144, 57 and 111, respectively. Even though the amount of water present in these systems appears to be low, the mole ratios of water to copolymer are quite large owing to the high molecular weight of P123. Quantum yields and lifetimes of DODCI have been measured in these reverse phases over the temperature range $293-318 \mathrm{~K}$. Figure 11 gives plots of $\phi_{f}$ vs $T$ for DODCI in $L_{2},{ }^{\mathrm{I}} L_{2}$ and $I_{2}$ as a function of temperature and they are almost identical in the three systems at a given temperature. The figure also displays quantum yield values of the probe in water and butyl acetate for comparison. It can be noticed from the figure that the quantum yields of DODCI are significantly lower in water and butyl acetate compared to the reverse micellar phases. Fluorescence decays of DODCI in these systems exhibit bi-exponential behaviour with two lifetimes ( $\tau_{f 1}$ and $\tau_{f 2}$ ) and the contribution of $\tau_{f 2}$ is only $10 \%-15 \%$.

The interesting observation from these measurements, however, is the presence of $10 \%-15 \%$ short component whose values match with those obtained in water, which is an indication that water present in the interiors of $L_{2},{ }^{\mathrm{I}} L_{2}$, and $I_{2}$ phases of $\mathrm{P} 123$, is in the form of droplets and has the properties of bulk

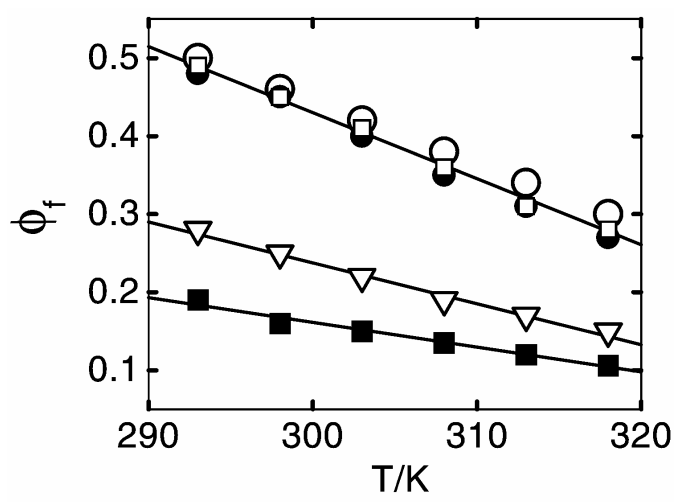

Figure 11. Plots of quantum yields vs $T$ for DODCI in water $(\nabla)$, butyl acetate $(\boldsymbol{\square}), L_{2}(\mathrm{O}),{ }^{\mathrm{I}} L_{2}(\boldsymbol{O})$ and $I_{2}(\square)$. The lines passing through the data points have been obtained by linear least-squares fit. Partially modified and reprinted with permission from ref. [29]. C) (2006) American Chemical Society. 
water. Although evidence for the existence of water droplets resembling the properties of bulk water in the cores of P123-butyl acetate-water reverse micellar systems has been obtained from this study, no indication can be given regarding the size of the droplets from these measurements. At this juncture it would be worthwhile to compare somewhat similar results obtained by Schelly and co-workers ${ }^{84}$ and Qi and $\mathrm{Ma}^{87}$ in case of aqueous reverse micelles formed with TX-100/benzene-hexane $(30: 70 \mathrm{v} / \mathrm{v})$ and TX-100/n-hexanol/cyclohexane, respectively. Schelly and co-workers ${ }^{84}$ have measured the shift in the absorption maxima $\left(\lambda_{\max }\right)$ of the probe 1-methyl8 -oxyquinolinium as a function of $W$ and found that even at $W=9$, the properties of the water droplet in the core are not similar to that of bulk water. They attribute this observation to the size of the water droplet, which is too small to have bulk properties. In contrast, Qi and $\mathrm{Ma}^{87}$ have measured the shift in $\lambda_{\max }$ of the probe methylene blue in aqueous TX100/n-hexanol/cyclohexane reverse micellar system with increasing $W$ and observed that at $W=5 \cdot 3$, the properties of the water droplet resemble that of bulk water. Although the property that has been monitored by us is different, our results are similar to the ones obtained by Qi and $\mathrm{Ma}^{87}$ The water droplet properties at three different values of $W(144,57$, and 111 in $L_{2},{ }^{\mathrm{I}} L_{2}$ and $I_{2}$, respectively) are identical to that of bulk water, which is evident from the similarities in the lifetime $\left(\tau_{f 2}\right)$ values of DODCI with the ones measured in water. This result indicates that the sizes of the water droplets present in the reverse phases of P123, even at the lowest value of $W$ used in this study, are probably large enough to possess the properties of bulk water. In a recent study, Fayer and co-workers ${ }^{88}$ have examined the properties of water droplet present in the reverse micelles formed with anionic surfactant aerosol-OT (AOT) in isooctane using linear and nonlinear infrared spectroscopy and found that at $W>20$ (corresponding to a water pool diameter of $7.0 \mathrm{~nm}$ ), the water pool in AOT reverse micelles displays the properties of bulk water. However, it must be noted that the linear relationship, which exists between $W$ and water-pool diameter in AOT reverse micelles does not hold in case of nonionic systems. In the context of these investigations, where the main emphasis has been to examine the nature of the water trapped in nano confinements, the result obtained by us in a way contributes to the understanding of the interiors of the reverse phases of the block copolymer systems, which are virtually unexplored.
Now turning our attention to the photoisomerization of DODCI in the reverse phases of P123, which is the second objective of this work, the nonradiative and radiative rate constants have been calculated from the measured quantum yields and lifetimes as mentioned earlier. Figure 12 displays plots of $\ln \left(k_{n r}\right)$ vs $1 / T$ of DODCI in $L_{2},{ }^{\mathrm{I}} L_{2}$ and $I_{2}$ over the temperature range $293-318 \mathrm{~K}$. The data for water and butyl acetate are also given in the figure for comparison. It is evident from the figure that the isomerization rate constants of DODCI at a particular temperature are identical for the three values of $W$ used in this study but are significantly lower compared to water and butyl acetate. The reasons for the observed behaviour are as follows.

In nonionic reverse micellar systems, the added water initially hydrates the polyethylene oxide chains and the formation of water droplet transpires only when these chains are completely hydrated. It may be recalled that in $L_{2},{ }^{\mathrm{I}} L_{2}$, and $I_{2}$, the $W$ values are 144,57 , and 111, respectively, and water droplets exist in each of the three samples, which means that the hydration of polyethylene oxide chains is complete. Since a major fraction of DODCI is solubilized in the core region where hydrated polyethylene oxide chains are present, it experiences an identical microenvironment in the three reverse phases, thus the photoisomerization rate constants are the same. In such a scenario, the logical question, which arises is, what happens to the photoisomerization rate constant if the polyethylene oxide chains are not hydrated? In other words, how the isomerization of

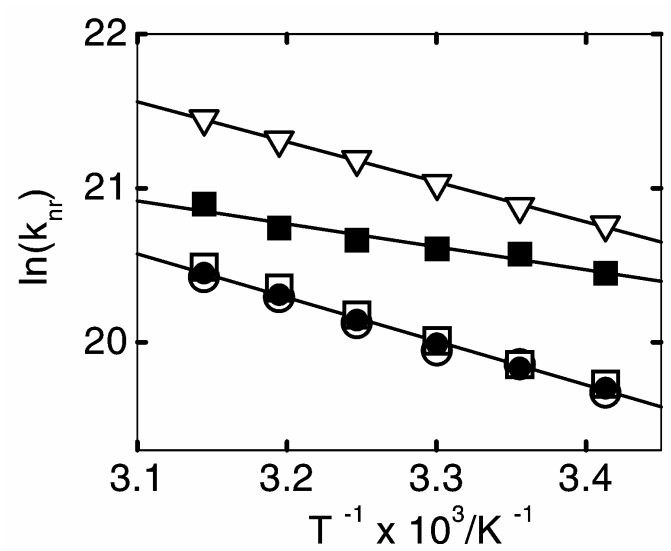

Figure 12. Plots of $\ln \left(k_{n r}\right)$ vs $1 / T$ for DODCI in water $(\nabla)$, butyl acetate $(\square), L_{2}(\bigcirc),{ }^{\mathrm{I}} L_{2}(\mathbf{O})$ and $I_{2}(\square)$. The lines passing through the data points have been obtained by linear least-squares fit. Partially modified and reprinted with permission from ref. [29]. C (2006) American Chemical Society. 
DODCI would be affected in the absence of water in these reverse phases. We have made an attempt to address this issue by making $L_{2}$ phase with $14 \%$ P123, 86\% butyl acetate and without water. Unfortunately, no measurements could be performed in this system as the solute DODCI degraded instantaneously upon dissolving. To find out how the photoisomerization rate constants of DODCI in these reverse phases measure up to the ones obtained in normal phases of $\mathrm{P} 123$, data from figures 10 and 12 have been compared. Such comparison reveals that the isomerization rate constants are almost similar in normal and reverse phases, which is not surprising considering the fact that in normal phases, the site of solubilization of DODCI is the palisade layer, whereas it is situated in the core region in the reverse phases and both these locales constitute hydrated polyethylene oxide chains. The marginally higher rate constants obtained in case of the reverse phases is probably due to the penetration of butyl acetate inside the cores of the reverse phases, which increases the fluidity of the surroundings.

\section{Summary and outlook}

In this work, an attempt has been made to explore the interiors of four different phases (normal micellar, normal micellar cubic, reverse micellar and reverse micellar cubic phases) formed by a typical triblock copolymer, P123. This has been achieved by monitoring processes such as rotational diffusion and photoisomerization of organic solutes solubilized in these polymorphic environments. The important conclusions from this study and the direction in which we plan to carry out our future work are discussed in this section. It has been noticed that fluorescence anisotropy measurements are sensitive to unimer-micelle transition as the solute molecules experience different environments in both these phases of the block copolymer. In contrast, it has become evident from our work that such measurements carried out with hydrophobic probes do not discern sol-gel transition, since the probes experience an identical environment in both these phases. Efforts have already begun to find out whether solgel transition can be monitored by carrying out investigations with ionic solutes, which can be solubilized at the micelle-water interface, because the microenvironment in this region is altered significantly upon gelation. Photoisomerization studies carried out in $L_{1}$ and $I_{1}$ phases of P123 complement the rotational diffusion measurements, as the solute used for this purpose DODCI, is also solubilized in an identical environment in both the phases. Similar studies carried out in the reverse phases $\left(L_{2}\right.$ and $\left.I_{2}\right)$ indicate that the water present in these systems is in the form of water droplets and has the properties of bulk water. Our future plan is to extend these studies to systems such as surfactant-copolymer micelles and gels to get a better appreciation of dynamical processes in more complex yet 'interesting' environments.

\section{Acknowledgments}

We are grateful to Dr Rajib Ganguly for many useful discussions on block copolymers systems and also the providing us with relevant literature. We also acknowledge Drs S K Sarkar and T Mukherjee for their encouragement throughout the course of this work.

\section{References}

1. Zhou Z and Chu B 1988 J. Colloid. Interface Sci. 126171

2. Almgren M, van Stam J, Lindblad C, Li P, Stilbs P and Bahadur P 1991 J. Phys. Chem. 955677

3. Mortensen K, Brown W and Nordén B 1992 Phys. Rev. Lett. 862340

4. Linse P and Malmsten M 1992 Macromolecules 255434

5. Mortensen K and Linse P 1992 Macromolecules 255440

6. Mortensen K and Pedersen J S 1993 Macromolecules 26805

7. Alexandridis P, Holzwarth J F and Hatton T A 1994 Macromolecules 272414

8. Wanka G, Hoffmann H and Ulbricht W 1994 Macromolecules 274145

9. Zhang K and Khan A 1995 Macromolecules 283807

10. Alexandridis P, Olsson U and Lindman B 1995 Macromolecules $\mathbf{2 8} 7700$

11. Chu B 1995 Langmuir 11414

12. Alexandridis P, Zhou D and Khan A 1996 Langmuir 122690

13. Chu B and Zhou Z 1996 In Nonionic surfactants (ed.) V M Nace, Surfactant Science Series (New York: Marcel Dekker) vol. 60, p. 67, and references therein

14. Holmqvist P, Alexandridis P and Lindman B $1998 \mathrm{~J}$. Phys. Chem. B102 1149

15. Jansson J, Schillén K, Olofsson G, da Silva R C and Loh W 2004 J. Phys. Chem. B108 82

16. Jansson J, Schillén K, Nilsson M, Söderman O, Fritz G, Bergmann A and Glatter O 2005 J. Phys. Chem. B109 7073

17. Ganguly R, Aswal V K, Hassan P A, Gopalakrishnan I K and Yakhmi J V 2005 J. Phys. Chem. B109 5653

18. Ganguly R, Aswal V K, Hassan P A, Gopalakrishnan I K and Kulshreshtha S K 2006 J. Phys. Chem. B110 9843 
19. Jeon S, Granick S, Kwon K C and Char K 2002 J. Polym. Sci. B40 2883

20. Dutt G B 2005 J. Phys. Chem. B109 4923

21. Mali K S, Dutt G B, Ganguly R and Mukherjee T 2005 J. Chem. Phys. 123144913

22. Mali K S, Dutt G B and Mukherjee T 2007 Langmuir 231041

23. Grant C D, DeRitter M R, Steege K E, Fadeeva T A and Castner E W Jr 2005 Langmuir 211745

24. Grant C D, Steege K E, Bunagan M R, Castner E W Jr 2005 J. Phys. Chem. B109 22273

25. Sen P, Ghosh S, Sahu K, Mondal S K, Roy D and Bhattacharyya K 2006 J. Chem. Phys. 124204905

26. Kumbhakar M, Goel T, Nath S, Mukherjee T and Pal H 2006 J. Phys. Chem. B110 25646

27. Mali K S, Dutt G B and Mukherjee T 2006 J. Chem. Phys. 124054904

28. Mali K S, Dutt G B and Mukherjee T 2006 J. Chem. Phys. 124199901

29. Mali K S, Dutt G B and Mukherjee T 2006 Langmuir 226837

30. Dutt G B 2005 ChemPhysChem 6 413, and references therein

31. Waldeck D H 2000 In Conformational analysis of molecules in the excited state (ed.) J Waluk (New York: Wiley-VCH) p. 113, and references therein

32. Quitevis E L, Marcus A H and Fayer M D $1993 \mathrm{~J}$. Phys. Chem. 975762

33. Maiti N C, Krishna M M G, Britto P J and Periasamy N 1997 J. Phys. Chem. B101 11051

34. Krishna M M G, Das R and Periasamy N $2000 \mathrm{~J}$. Chem. Phys. 1128502

35. Dutt G B 2002 J. Phys. Chem. B106 7398

36. Dutt G B 2003 J. Phys. Chem. B107 3131

37. Dutt G B 2003 J. Phys. Chem. B107 10546

38. Dutt G B 2004 J. Phys. Chem. B108 3651

39. Dutt G B 2005 Langmuir 2110391

40. Kelepouris L and Blanchard G J 2003 J. Phys. Chem. B107 1079

41. Wittouck N, Negri R M, Ameloot M and De Schryver F C 1994 J. Am. Chem. Soc. 11610601

42. Dutt G B 2004 J. Phys. Chem. B108 805

43. Dutt G B 2004 J. Phys. Chem. B108 7944

44. Mali K S, Dutt G B and Mukherjee T 2006 J. Chem. Phys. 123174504

45. Debye P 1929 Polar molecules (New York: Dover)

46. $\mathrm{Hu} \mathrm{C} \mathrm{M}$ and Zwanzig R 1974 J. Chem. Phys. 604354

47. Dutt G B, Srivatsavoy V J P and Sapre A V 1999 J. Chem. Phys. 1109623

48. Dutt G B and Sachdeva A 2003 J. Chem. Phys. 1188307

49. Almgren M, Grieser F and Thomas J K $1979 \mathrm{~J}$. Am Chem. Soc. 101279

50. Mukerjee P and Cardinal J R 1978 J. Phys. Chem. 82 1620

51. Mukerjee P 1979 In Solution chemistry of surfactants (ed.) K L Mittal (New York: Plenum) vol. 1

52. Ganesh K N, Mitra P and Balasubramanian D $1982 \mathrm{~J}$. Phys. Chem. 864291

53. Kinoshita J, Kawato S and Ikegami A 1977 Biophys. J. 20289
54. Lipari G and Szabo A 1980 Biophys. J. 30489

55. Lipari G and Szabo A 1982 J. Am. Chem. Soc. 104 4546

56. Wang C C and Pecora R 1980 J. Chem. Phys. 725333

57. Prazeres T J V, Fedorov A and Martinho J M G 2004 J. Phys. Chem. B108 9032

58. Sen S, Sukul D, Dutta P and Bhattacharyya K $2001 \mathrm{~J}$. Phys. Chem. A105 7495

59. Fleming G R and Wolynes P G 1990 Phys. Today 36

60. Waldeck D H 1991 Chem. Rev. 91 415, and references therein

61. Kramers H A 1940 Physica 7284

62. Velsko S P and Fleming G R 1982 J. Chem. Phys. 76 3553

63. Keery K M and Fleming G R 1982 Chem. Phys. Lett. 93322

64. Lee M, Bain A J, McCarthy P J, Han C H, Haseltine J N, Smith A B III and Hochstrasser R M $1986 \mathrm{~J}$. Chem. Phys. 854341

65. Lee M, Haseltine J N, Smith A B and Hochstrasser R M 1989 J. Am. Chem. Soc. 1115044

66. Anderton R M and Kauffman J F 1994 J. Phys. Chem. 9812125

67. Anderton R M and Kauffman J F 1995 J. Phys. Chem. 9914628

68. Rullière C 1976 Chem. Phys. Lett. 43303

69. Sundström V and Gillbro T 1982 J. Phys. Chem. 86 1788

70. Velsko S P and Fleming G R 1982 Chem. Phys. 6559

71. Velsko S P, Waldeck D H and Fleming G R $1983 J$. Chem. Phys. 78249

72. Hara K and Akimoto S 1991 J. Phys. Chem. 955811

73. Aramendía P F, Negri R M and Román E S $1994 \mathrm{~J}$. Phys. Chem. 983165

74. Sitzmann E V and Eisenthal K B 1988 J. Phys. Chem. 924579

75. Shi X, Borguet E, Tarnosky A N and Eisenthal K B 1996 Chem. Phys. 205167

76. Datta A, Mandal D, Pal S K and Battacharyya K 1997 Chem. Phys. Lett. 27877

77. Pal S K, Datta A, Mandal D and Battacharyya K 1998 Chem. Phys. Lett. $\mathbf{2 8 8} 793$

78. Streletzky K and Phillies G D J 1995 Langmuir 1142

79. Molina-Bolívar J A, Aguiar J and Ruiz C C $2002 \mathrm{~J}$. Phys. Chem. B106 870

80. Lackowicz J R 1983 Principles of fluorescence spectroscopy (New York: Plenum)

81. Zhu D-M, Feng K-I and Schelly Z A 1992 J. Phys. Chem. 962382

82. Zhu D-M and Schelly Z A 1992 Langmuir 848

83. Zhu D-M, Wu X and Schelly Z A 1992 Langmuir 81538

84. Zhu D-M, Wu X and Schelly Z A 1992 J. Phys. Chem. 967121

85. Almgren M, van Stam J, Swarup S and Löfroth J-E 1986 Langmuir 2432

86. Rodríguez R, Vargas S and Fernández-Velasco D A 1998 J. Colloid Interface Sci. 19721

87. Qi L and Ma J 1998 J. Colloid Interface Sci. 19736

88. Piletic I R, Moilanen D E, Spry D B, Levinger N E and Fayer M D 2006 J. Phys. Chem. A110 4985 